Supporting Information for:

\title{
Controllable Syntheses, Crystal Structure Evolution and Photoluminescence of Polymorphic Zirconium Oxyfluorides
}

Chen Li, Ting Wen, Ke Liu, Dequan Jiang, Zimin Jiang, and Yonggang Wang* Center for High Pressure Science and Technology Advanced Research (HPSTAR), Beijing 100094, China

*E-mail: yonggang.wang@hpstar.ac.cn

Figure S1. The powder XRD patterns of samples synthesized with dry Ar flow under various conditions of reaction at (a) $500{ }^{\circ} \mathrm{C}$ for $3 \mathrm{~h}$; (b) $600{ }^{\circ} \mathrm{C}$ for $3 \mathrm{~h}$; (c) $700{ }^{\circ} \mathrm{C}$ for $3 \mathrm{~h}$; (d) $750{ }^{\circ} \mathrm{C}$ for $3 \mathrm{~h}$; (e) $750{ }^{\circ} \mathrm{C}$ for $2 \mathrm{~h}$.

Figure S2. Rietveld refinements of $\mathrm{ZrO}_{0.33} \mathrm{~F}_{3.33}$ phase synthesized in this work based on (a) the structure with space group $P 4 / \mathrm{mbm}, R_{\mathrm{p}}=3.21 \%, R_{\mathrm{wp}}=6.73 \%$; (b) the structure of tetragonal $\mathrm{ZrF}_{4}$ with space group $P 4_{2} / m, R_{\mathrm{p}}=2.75 \%, R_{\mathrm{wp}}=5.83 \%$ (Red arrows: unindexed diffraction peaks).

Figure S3. (a) FWHM of the recognizable diffraction peaks at $2 \theta<80^{\circ}$ in the experimental powder XRD data of $\mathrm{ZrO}_{0.33} \mathrm{~F}_{3.33}$; (b) the experimental and simulated patterns of diffraction peaks at $35.6^{\circ}, 37.4^{\circ}$ and $39.2^{\circ}$ of $\mathrm{ZrO}_{0.33} \mathrm{~F}_{3.33}$.

Figure S4. LeBail fitting results of on the powder XRD patterns of mixed phases. (a) the phase containing $\mathrm{ZrO}_{0.46} \mathrm{~F}_{3.08}, R_{\mathrm{p}}=5.06 \%, R_{\mathrm{wp}}=7.94 \%$; (b) the phase containing $\mathrm{Zr}_{3} \mathrm{O}_{2} \mathrm{~F}_{8}, R_{\mathrm{p}}=4.20 \%$, $R_{\mathrm{wp}}=6.77 \%$; (c) the phase containing $\mathrm{ZrF}_{4}, R_{\mathrm{p}}=6.79 \%, R_{\mathrm{wp}}=9.56 \%$.

Figure S5. The powder XRD patterns of Ti-doped zirconia or zirconium oxyfluorides.

Figure S6. The photographs of Ti-doped zirconia or zirconium oxyfluorides under $254 \mathrm{~nm}$ UV lamp.

Table S1. Crystallographic data for $\mathrm{Zr}_{7} \mathrm{O}_{9} \mathrm{~F}_{10}$ and $\mathrm{ZrO}_{0.33} \mathrm{~F}_{3.33}$ (Powder).

Table S2. The refined atomic parameters of $\mathrm{Zr}_{7} \mathrm{O}_{9} \mathrm{~F}_{10}$.

Table S3. The refined atomic parameters of $\mathrm{ZrO}_{0.33} \mathrm{~F}_{3.33}$. 

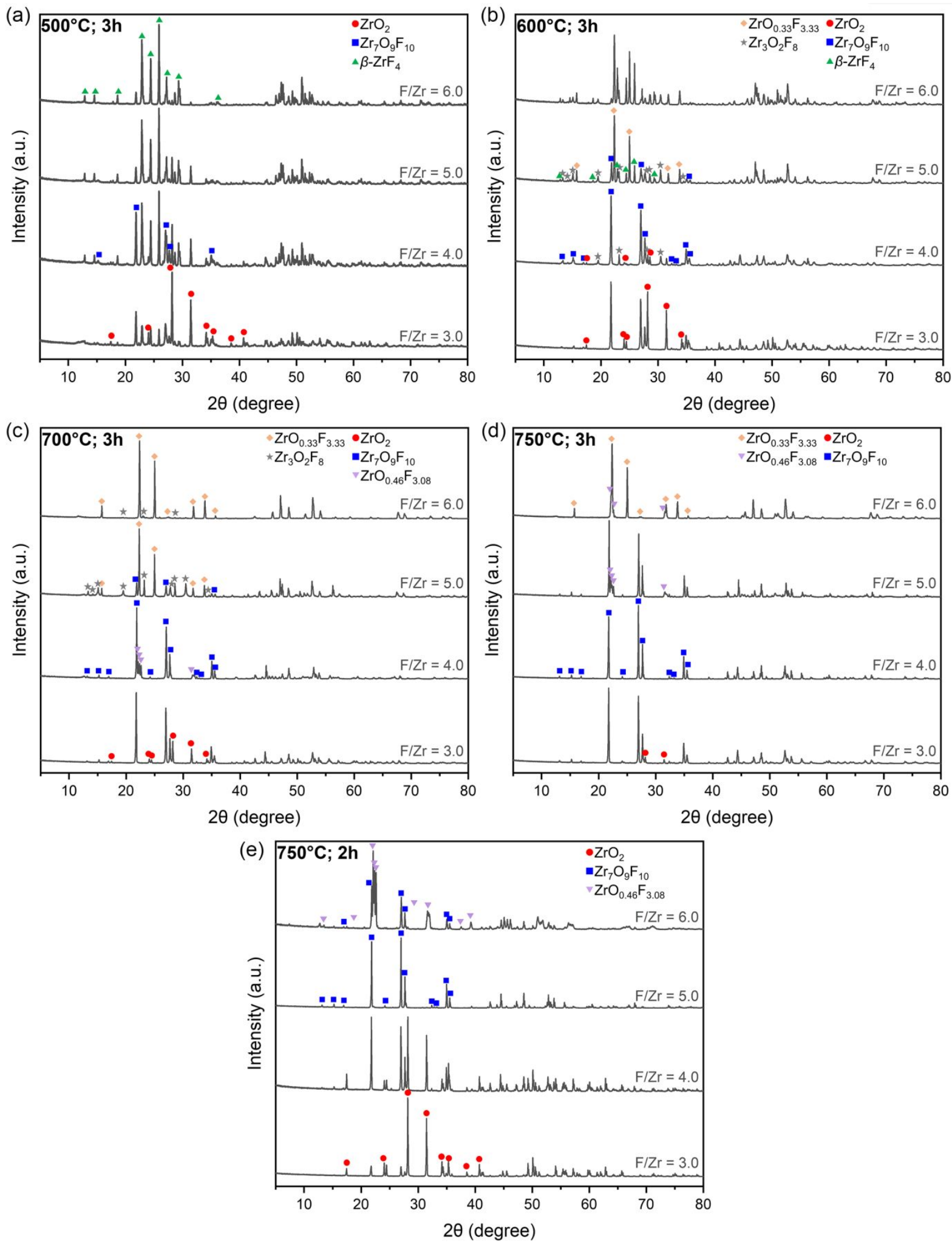

Figure S1. The powder XRD patterns of samples synthesized with dry Ar flow under various conditions of reaction at (a) $500{ }^{\circ} \mathrm{C}$ for $3 \mathrm{~h}$; (b) $600{ }^{\circ} \mathrm{C}$ for $3 \mathrm{~h}$; (c) $700{ }^{\circ} \mathrm{C}$ for $3 \mathrm{~h}$; (d) $750{ }^{\circ} \mathrm{C}$ for $3 \mathrm{~h}$; (e) $750{ }^{\circ} \mathrm{C}$ for $2 \mathrm{~h}$. 

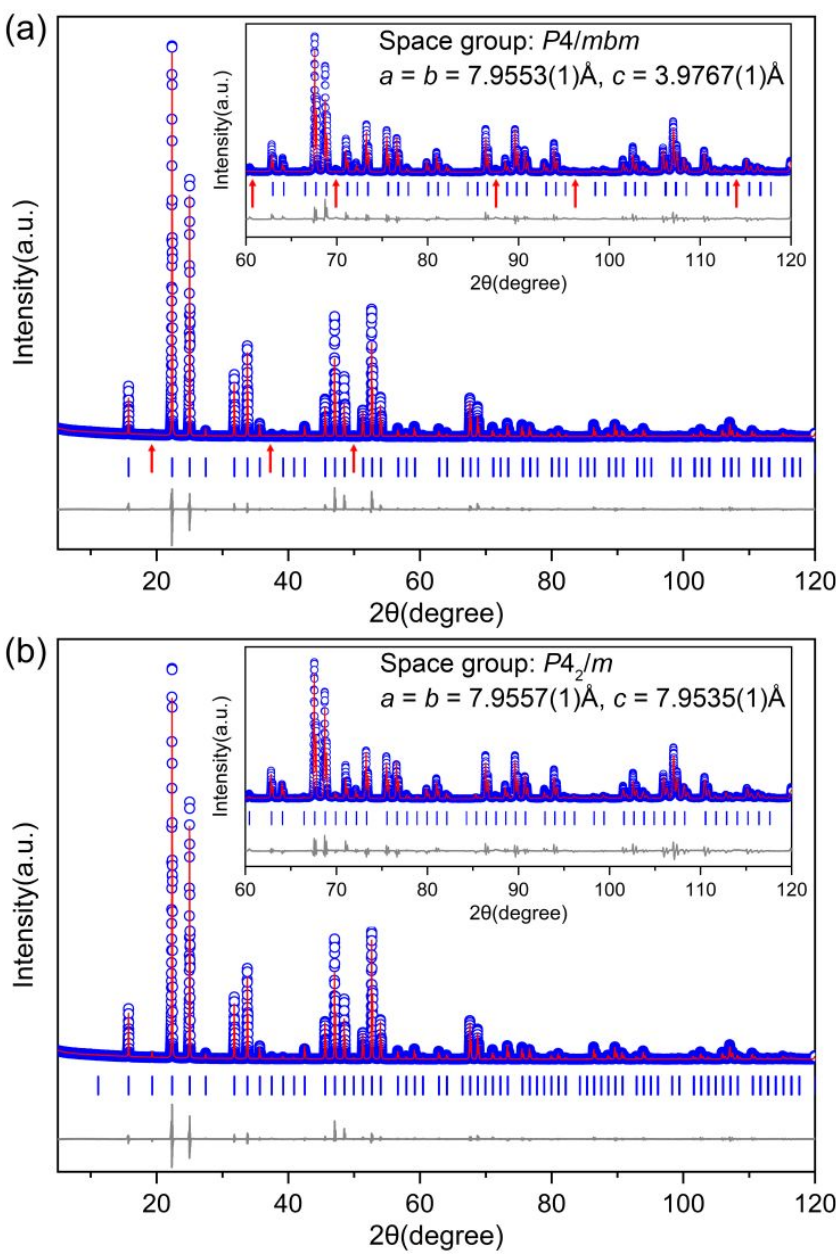

Figure S2. Rietveld refinements of $\mathrm{ZrO}_{0.33} \mathrm{~F}_{3.33}$ phase synthesized in this work based on (a) the structure with space group $P 4 / \mathrm{mbm}, R_{\mathrm{p}}=3.21 \%, R_{\mathrm{wp}}=6.73 \%$; (b) the structure of tetragonal $\mathrm{ZrF}_{4}$ with space group $P 4_{2} / m, R_{\mathrm{p}}=2.75 \%, R_{\mathrm{wp}}=5.83 \%$ (Red arrows: unindexed diffraction peaks).
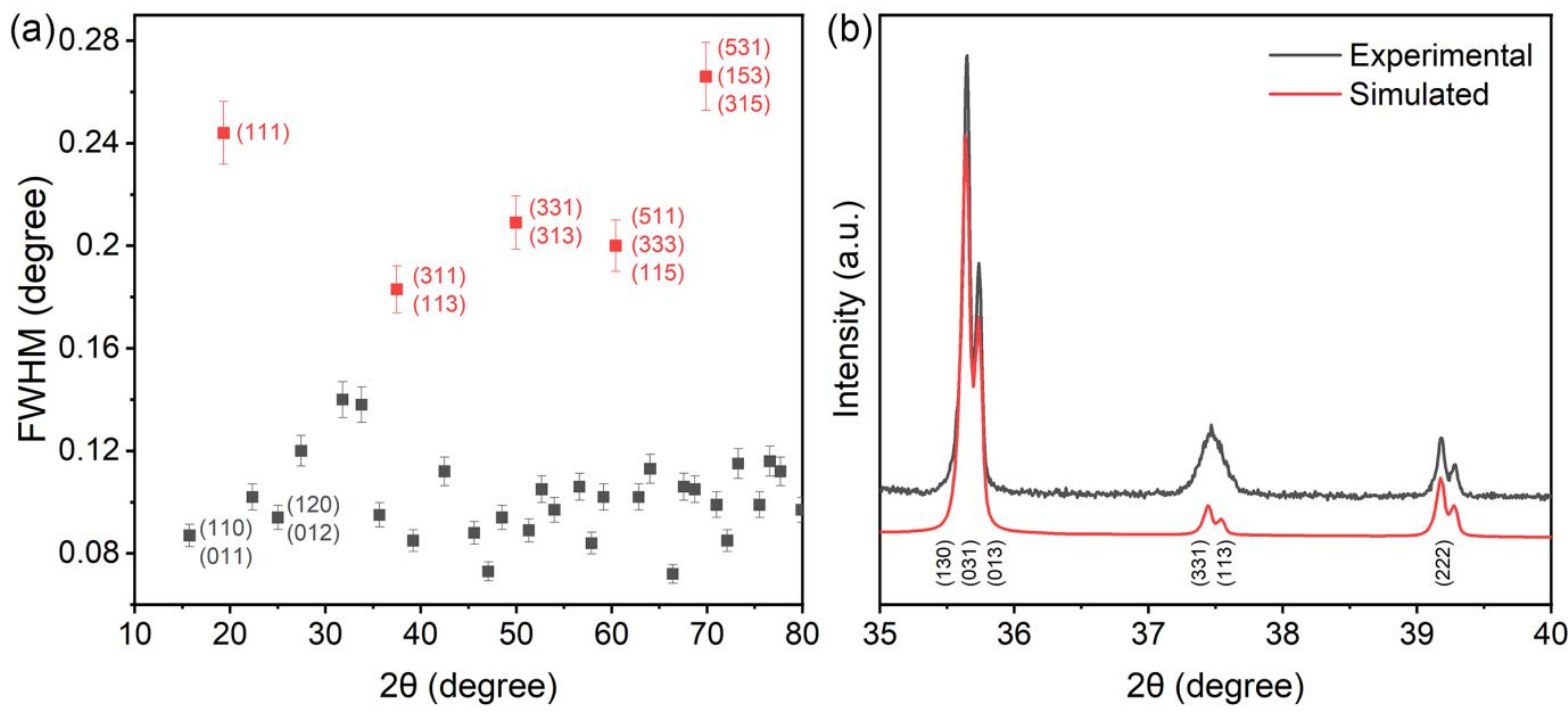

Figure S3. (a) FWHM of the recognizable diffraction peaks at $2 \theta<80^{\circ}$ in the experimental powder $\mathrm{XRD}$ data of $\mathrm{ZrO}_{0.33} \mathrm{~F}_{3.33}$; (b) the experimental and simulated patterns of diffraction peaks at $35.6^{\circ}, 37.4^{\circ}$ and $39.2^{\circ}$ of $\mathrm{ZrO}_{0.33} \mathrm{~F}_{3.33}$. 

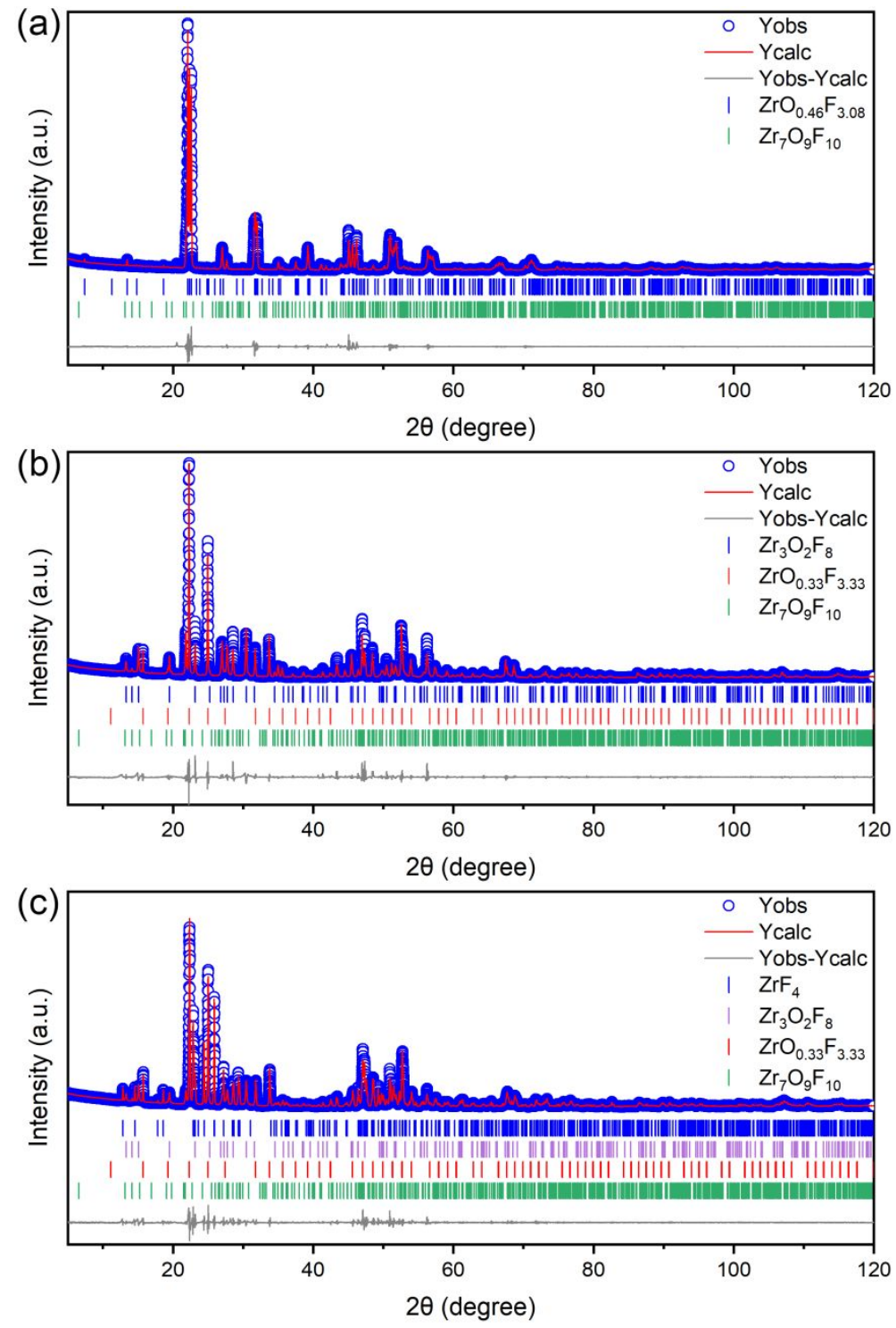

Figure S4. LeBail fitting results of on the powder XRD patterns of mixed phases. (a) the phase containing $\mathrm{ZrO}_{0.46} \mathrm{~F}_{3.08}, R_{\mathrm{p}}=5.06 \%, R_{\text {wp }}=7.94 \%$; (b) the phase containing $\mathrm{Zr}_{3} \mathrm{O}_{2} \mathrm{~F}_{8}, R_{\mathrm{p}}=4.20 \%, R_{\text {wp }}=$ $6.77 \%$; (c) the phase containing $\mathrm{ZrF}_{4}, R_{\mathrm{p}}=6.79 \%, R_{\mathrm{wp}}=9.56 \%$. 


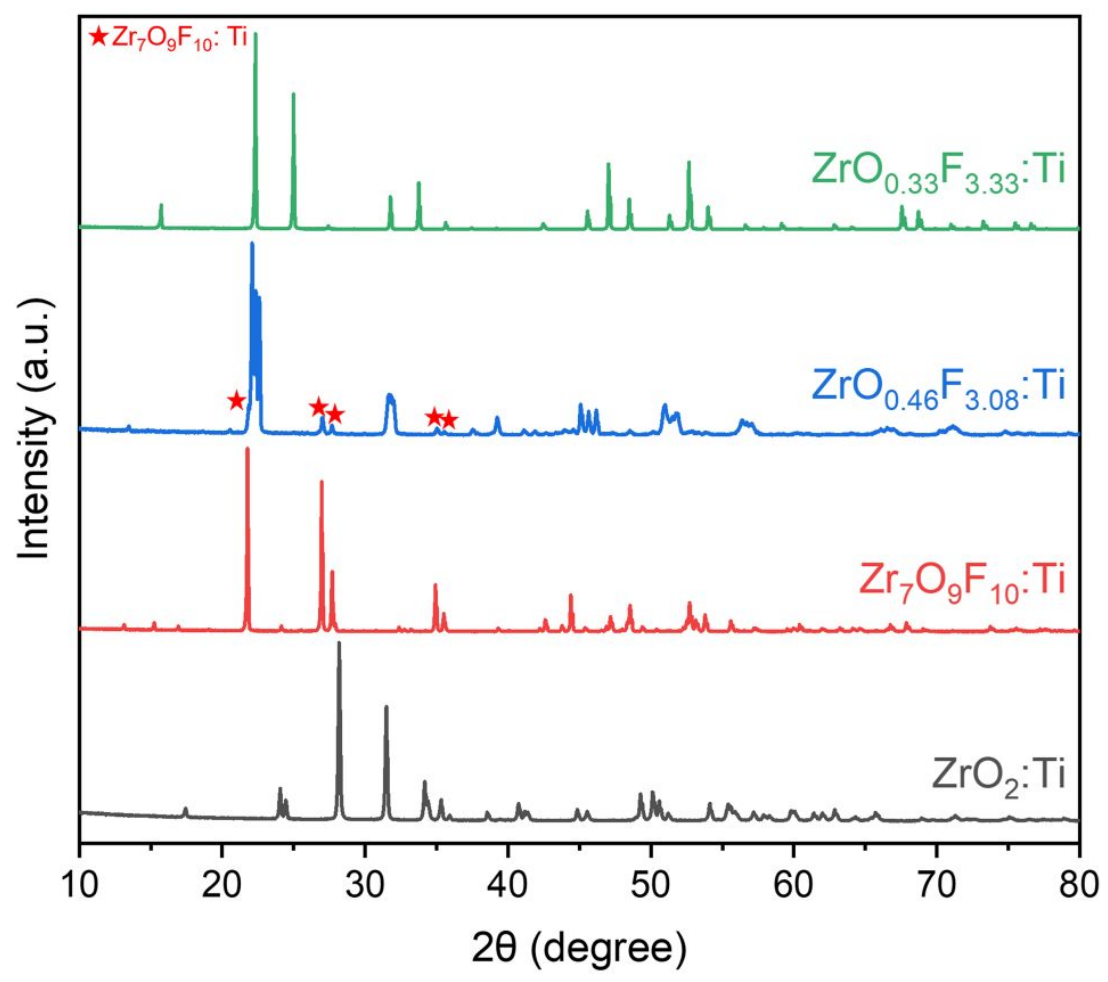

Figure S5. The powder XRD patterns of Ti-doped zirconia or zirconium oxyfluorides.
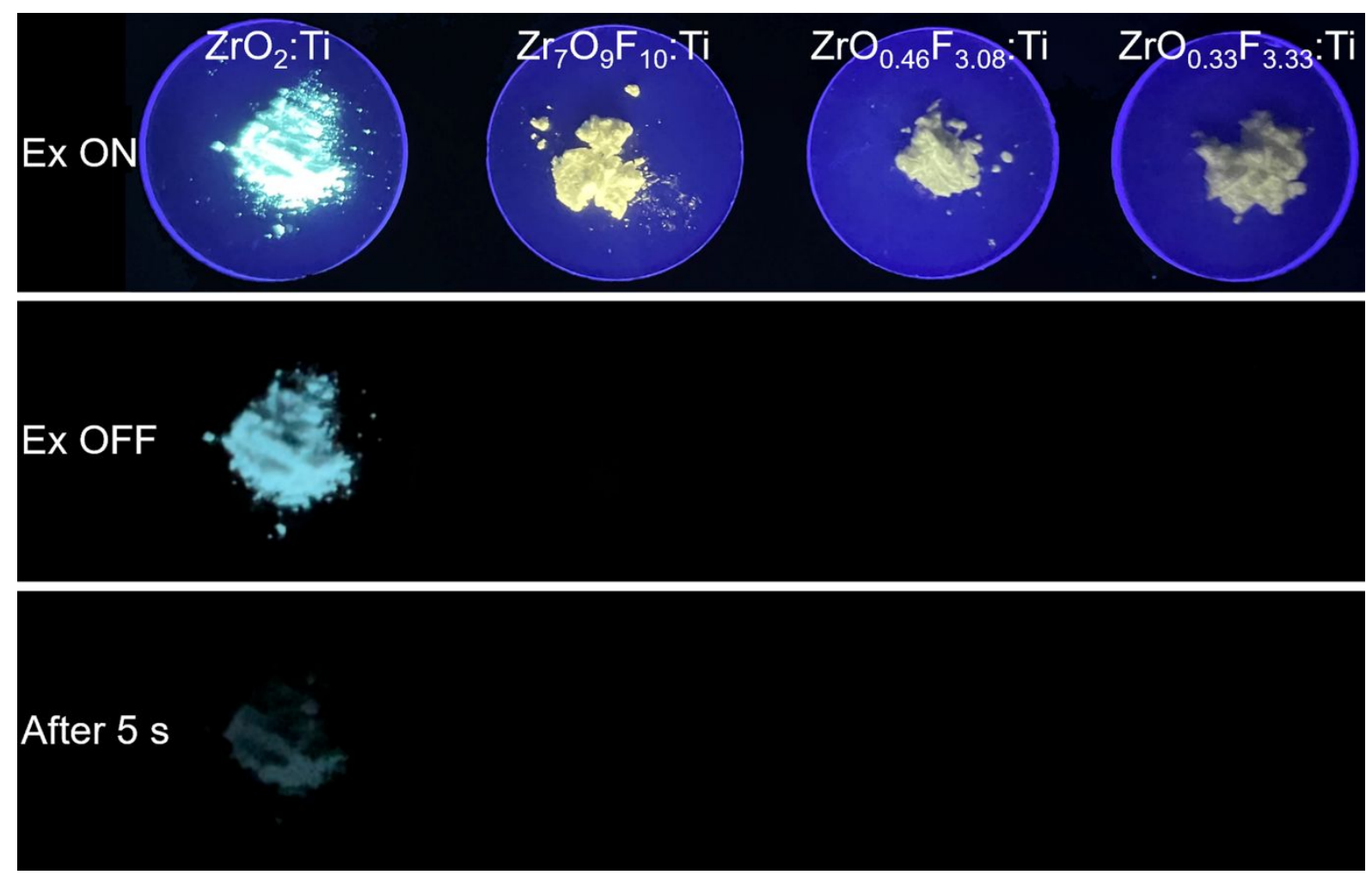

Figure S6. The photographs of Ti-doped zirconia or zirconium oxyfluorides under $254 \mathrm{~nm}$ UV lamp. 
Table S1. Crystallographic data for $\mathrm{Zr}_{7} \mathrm{O}_{9} \mathrm{~F}_{10}$ and $\mathrm{ZrO}_{0.33} \mathrm{~F}_{3.33}$ (Powder).

\begin{tabular}{|c|c|c|}
\hline Formula & $\mathrm{Zr}_{7} \mathrm{O}_{9} \mathrm{~F}_{10}$ & $\mathrm{ZrO}_{0.33} \mathrm{~F}_{3.33}$ \\
\hline Formula weight & 972.55 & 159.77 \\
\hline Temperature (K) & 293(2) & $293(2)$ \\
\hline Crystal system & Orthorhombic & Tetragonal \\
\hline Space group (No.) & Pbam (No. 55) & $P 4_{2} / m$ (No. 84$)$ \\
\hline$a(\AA)$ & $6.4331(1)$ & $7.9558(1)$ \\
\hline$b(\AA)$ & $26.9344(2)$ & $7.9558(1)$ \\
\hline$c(\AA)$ & $4.0755(1)$ & $7.9535(1)$ \\
\hline$\alpha(\mathrm{deg})$ & 90 & 90 \\
\hline$\beta(\mathrm{deg})$ & 90 & 90 \\
\hline$\gamma(\operatorname{deg})$ & 90 & 90 \\
\hline$V\left(\AA^{3}\right)$ & $706.17(1)$ & $503.41(1)$ \\
\hline$Z$ & 2 & 8 \\
\hline Radiation & $\operatorname{CuK} \alpha(\lambda=1.5406 \AA)$ & $\operatorname{CuK} \alpha(\lambda=1.5406 \AA)$ \\
\hline$d$-space range $(\AA)$ & $0.8895-17.676$ & $0.8895-17.676$ \\
\hline$R_{\mathrm{p}}^{a}(\%)$ & 2.44 & 2.75 \\
\hline$R_{\mathrm{wp}}^{b}(\%)$ & 4.33 & 5.83 \\
\hline
\end{tabular}


Table S2. The refined atomic parameters of $\mathrm{Zr}_{7} \mathrm{O}_{9} \mathrm{~F}_{10}$.

\begin{tabular}{|c|c|c|c|c|c|c|}
\hline Atoms & Site & $x$ & $y$ & $z$ & Occ. & $B_{e q}$ \\
\hline $\mathrm{Zr} 1$ & $2 a$ & 0 & 0 & 0 & 1 & $1.60(1)$ \\
\hline $\mathrm{Zr} 2$ & $4 g$ & $0.4475(1)$ & $0.0625(1)$ & 0 & 1 & $1.12(1)$ \\
\hline $\mathrm{Zr} 3$ & $4 g$ & $0.0039(1)$ & $0.1469(1)$ & 0 & 1 & $0.72(1)$ \\
\hline $\mathrm{Zr} 4$ & $4 g$ & $0.4548(1)$ & $0.2218(1)$ & 0 & 1 & $1.78(1)$ \\
\hline $\mathrm{O} 1$ & $4 g$ & $0.7068(2)$ & $0.0095(1)$ & 0 & 0.474 & $1.74(1)$ \\
\hline $\mathrm{F} 1$ & $4 g$ & $0.7068(2)$ & $0.0095(1)$ & 0 & 0.526 & $1.74(1)$ \\
\hline $\mathrm{O} 2$ & $4 g$ & $0.0963(2)$ & $0.0655(1)$ & 0 & 0.474 & $1.74(1)$ \\
\hline $\mathrm{F} 2$ & $4 g$ & $0.0963(2)$ & $0.0655(1)$ & 0 & 0.526 & $1.74(1)$ \\
\hline $\mathrm{O} 3$ & $4 g$ & $0.6988(2)$ & $0.1040(1)$ & 0 & 0.474 & $1.74(1)$ \\
\hline F3 & $4 g$ & $0.6988(2)$ & $0.1040(1)$ & 0 & 0.526 & $1.74(1)$ \\
\hline $\mathrm{O} 4$ & $4 g$ & $0.3665(2)$ & $0.1363(1)$ & 0 & 0.474 & $1.74(1)$ \\
\hline $\mathrm{F} 4$ & $4 g$ & $0.3665(2)$ & $0.1363(1)$ & 0 & 0.526 & $1.74(1)$ \\
\hline O5 & $4 g$ & $0.7883(2)$ & $0.1894(1)$ & 0 & 0.474 & $1.74(1)$ \\
\hline F5 & $4 g$ & $0.7883(2)$ & $0.1894(1)$ & 0 & 0.526 & $1.74(1)$ \\
\hline O6 & $4 g$ & $0.1454(2)$ & $0.2140(1)$ & 0 & 0.450 & $1.74(1)$ \\
\hline F6 & $4 g$ & $0.1454(2)$ & $0.2140(1)$ & 0 & 0.550 & $1.74(1)$ \\
\hline $\mathrm{O} 7$ & $2 b$ & 0 & 0 & 0.5 & 0.450 & $1.74(1)$ \\
\hline F7 & $2 b$ & 0 & 0 & 0.5 & 0.550 & $1.74(1)$ \\
\hline O8 & $4 h$ & $0.4633(3)$ & $0.0717(1)$ & 0.5 & 0.450 & $1.74(1)$ \\
\hline F8 & $4 h$ & $0.4633(3)$ & $0.0717(1)$ & 0.5 & 0.550 & $1.74(1)$ \\
\hline O9 & $4 h$ & $0.0079(2)$ & $0.1419(1)$ & 0.5 & 0.450 & $1.74(1)$ \\
\hline F9 & $4 h$ & $0.0079(2)$ & $0.1419(1)$ & 0.5 & 0.550 & $1.74(1)$ \\
\hline $\mathrm{O} 10$ & $4 h$ & $0.4430(2)$ & $0.2224(1)$ & 0.5 & 0.450 & $1.74(1)$ \\
\hline F10 & $4 h$ & $0.4430(2)$ & $0.2224(1)$ & 0.5 & 0.550 & $1.74(1)$ \\
\hline
\end{tabular}


Table S3. The refined atomic parameters of $\mathrm{ZrO}_{0.33} \mathrm{~F}_{3.33}$.

\begin{tabular}{lllllll}
\hline Atoms & Site & $x$ & $y$ & $z$ & Occ. & $B_{e q}$ \\
\hline Zr1 & $8 k$ & $0.1824(1)$ & $0.3202(1)$ & $0.2432(2)$ & 1 & $0.70(2)$ \\
O1 & $8 k$ & $0.7124(2)$ & $0.4240(2)$ & $0.2039(1)$ & 0.085 & $0.15(1)$ \\
F1 & $8 k$ & $0.7124(2)$ & $0.4240(2)$ & $0.2039(1)$ & 0.853 & $0.15(1)$ \\
O2 & $8 k$ & $0.1957(3)$ & $0.0434(2)$ & $0.2543(2)$ & 0.085 & $0.15(1)$ \\
F2 & $8 k$ & $0.1957(3)$ & $0.0434(2)$ & $0.2543(2)$ & 0.853 & $0.15(1)$ \\
O3 & $4 j$ & $0.7551(3)$ & $0.7559(3)$ & 0 & 0.081 & $0.15(1)$ \\
F3 & $4 j$ & $0.7551(3)$ & $0.7559(3)$ & 0 & 0.814 & $0.15(1)$ \\
O4 & $4 j$ & $0.6966(4)$ & $0.1884(4)$ & 0 & 0.081 & $0.15(1)$ \\
F4 & $4 j$ & $0.6966(4)$ & $0.1884(4)$ & 0 & 0.814 & $0.15(1)$ \\
O5 & $4 j$ & $0.1069(2)$ & $0.3586(2)$ & 0 & 0.081 & $0.15(1)$ \\
F5 & $4 j$ & $0.1069(2)$ & $0.3586(2)$ & 0 & 0.814 & $0.15(1)$ \\
O6 & $4 i$ & 0 & 0.5 & $0.3069(1)$ & 0.081 & $0.15(1)$ \\
F6 & $4 i$ & 0 & 0.5 & $0.3069(1)$ & 0.814 & $0.15(1)$ \\
\hline
\end{tabular}

